\title{
A comparison of yield from cervix versus vagina for culturing Candida albicans and Trichomonas vaginalis
}

\author{
A Joan P Boeke, Janny H Dekker, Paul G H Peerbooms
}

\begin{abstract}
Objective-To determine the agreement of culture results of Candida albicans and Trichomonas vaginalis from the cervix versus posterior fornix in women with vaginal symptoms.

Design-Same patient comparison of culture results from two sample sites.

Setting-Twenty one general practices in Amsterdam and the east of the Netherlands.

Subjects-Six hundred and eighty two women aged 15 to 55 years with vaginal symptoms, seen between 1 October 1987 and 31 May 1990.
\end{abstract}

Main outcome measures-For each site (cervix and posterior fornix) the proportion of detected $C$ albicans and $T$ vaginalis. The sensitivity of the cervical swab related to the vaginal one. The percentage of concordance for both microorganisms. Results-In 248 (34\%) women C albicans was diagnosed and in $38(6 \%) T$ vaginalis. In $99 \%$ of the proven $\boldsymbol{C}$ albicans cases, the yeast was found in the vagina. In $94 \% C$ albicans was isolated from the cervix. Sensitivity of the cervical swab was $94 \%$. In $98 \%$ of the patients a concordant observation was made regarding detection of yeast. In $97 \%$ of the proven $T$ vaginalis cases the protozoon was found in the vagina. In $91 \% T$ vaginalis was detected from the cervical swab. Sensitivity of the cervical swab was $92 \%$. The culture results were concordant in $99 \%$.

Conclusion-The yield from the vaginal source was slightly better than that from the cervix for culture of both microorganisms. For screening purposes, specimen-collection for culture of $N$ gonorrhoeae, $C$ albicans and $T$ vaginalis can be combined in one swab taken from the cervix.

(Genitourin Med 1993;69:41-43)

\section{Introduction}

Accurate diagnostic management of vaginal symptoms includes at least detection of Trichomonas vaginalis, Candida albicans, Neisseria gonorrhoeae and Chlamydia trachomatis. According to current medical practice among Dutch general practitioners this requires collection of three specimens: one from the cervix for culture of $N$ gonorrhoeae, a second one for cell culture of Chlamydia trachomatis and one from the vaginal mucosa for culture of
$C$ albicans and $T$ vaginalis.

As general practitioners, we were interested in reducing the number of samples needed to be taken. Although it is commonly advised to collect specimens from the vaginal mucosa for culture of $C$ albicans and $T$ vaginalis, both micro-organisms can also be found in the cervix. ${ }^{2}$ On the other hand, $N$ gonorrhoeae can only reliably be obtained from the cervix uteri. ${ }^{3}$ It would be useful from a practical and financial point of view to combine specimen collection from the cervix for culture of $N$ gonorrhoeae with that for $T$ vaginalis and $C$ albicans.

Culture is the gold standard for diagnosis of Candida albicans and Trichomonas vaginalis infections in cases of vaginitis. ${ }^{4-6}$ Genital $C$ albicans and $T$ vaginalis infections in women involve the squamous epithelium of the vagina. $C$ albicans and $T$ vaginalis adhere only to this type of epithelium. It is supposed that the columnar epithelium of the higher genital tract has a relative resistance against Trichomonas vaginalis. Consequently the vaginal mucosa is the source of choice for taking specimens for culture of $C$ albicans and $T$ vaginalis. ${ }^{47}$ However, little information is available from empirical research to support the diagnostic procedure mentioned. Therefore, we conducted a study which sought to answer the following question: What is the agreement of culture results of $C$ albicans and $T$ vaginalis from one cervical swab versus one swab from the posterior fornix in women with vaginal symptoms?

\section{Methods}

In 21 general practices in the Netherlands, all female patients between 15 and 55 years of age who consulted their doctor for vaginal symptoms between October 1987 and May 1990 were admitted to the study, after giving informed consent. Vaginal symptoms included itching, irritation or non bloody vaginal discharge of abnormal amount, smell or colour. The general practices were located in Amsterdam and an urbanised and rural area in the east of the Netherlands. The participating physicians were trained and instructed in advance concerning examination, specimen collection and administrative procedures. Each patient was subjected to a speculum examination of the vagina and specific note was made of the consistency, amount and colour of the vaginal discharge. Wet smears were examined by light microscope $(400 \times)$ for the presence of clue cells, leucocytes and motile trichomonads. 
The potassium hydroxide wet mount was examined for yeast pseudo-hyphae. The aminetest was done (fishy odour after addition of $\mathrm{KOH} 10 \%$ ) and the $\mathrm{pH}$ of the vaginal fluid was measured by means of a $\mathrm{pH}$ indicatorpaper (Specia).

One swab was taken from the cervix for culture of $N$ gonorrhoeae. This swab was reused for culture of $C$ albicans and $T$ vaginalis. A second specimen was collected from the posterior fornix of the vagina for culture of $C$ albicans and $T$ vaginalis. For collection of the cervical specimen the swab was inserted 1 or $2 \mathrm{~cm}$ into the external os and gently rotated for at least three seconds. An additional swab was taken from the cervix for an ELISA test of $C$ trachomatis. A special sample collection and transport kit was provided by the manufacturer of Chlamydiazyme ${ }^{r}$ (Abbott Laboratories). If the cervix was absent, the specimen collection was limited to the vagina.

\section{Microbiological methods}

The swabs were transported in Amies medium to the laboratory and processed within six hours after collection of the specimens. (Laboratory for Public Health, Municipal Health Service of Amsterdam or Laboratory for Microbiology and Immunology, Municipal Health Service Enschede).

The specimens collected by the Amsterdam practitioners were inoculated on chocolate agar and Thayer-Martin medium without antifungal agents. $N$ gonorrhoeae was recovered from both media, $C$ albicans and other yeasts from the second one mentioned. The specimens obtained by the practitioners outside Amsterdam were inoculated on Sabouraud medium for culturing $C$ albicans.

$T$ vaginalis was cultured by incubating the swabs in a cysteine-peptone-liver broth. ${ }^{8}$ All isolates were identified according to standard microbiological methods. ${ }^{9}$ The specimens obtained for the chlamydia ELISA test were sent to the microbiological laboratory of the OLVG Hospital (Amsterdam) on the day of collection for further processing. Within five days the ELISA was performed according to

Table 1 Frequencies of diagnoses in the study population (women with vaginal symptoms)

\begin{tabular}{lc}
\hline Diagnosis & $n(\%)$ \\
\hline Candida albicans & $250(37 \cdot 0)$ \\
Bacterial vaginosis & $122(18 \cdot 2)$ \\
Chlamydia trachomatis & $51(7 \cdot 7)$ \\
Trichomonas vaginalis & $39(5 \cdot 8)$ \\
Neisseria gonorrhoeae & $5(0 \cdot 7)$ \\
Double and triple infections total & $17(2 \cdot 5)$ \\
$C$ alb and $C$ trach & 9 \\
$C$ alb and $C$ trach and $N$ gon & 1 \\
$C$ alb and $T$ vag & 4 \\
$T$ vag and $C$ trach & 1 \\
$T$ vag and $N$ gon & 1 \\
$T$ vag and $C$ trach and $N$ gon & 1 \\
\hline
\end{tabular}

$C$ albicans and $T$ vaginalis were diagnosed if either the cervica or vaginal swab were positive in culture. Bacterial vaginosis was defined by three or four of the following criteria: Homogeneous discharge, aminetest positive, $\mathrm{pH}>\mathbf{4 . 5}$ or clue cells positive in absence of any specific infection.

( $\mathrm{n}=682$, missing: $C$ albicans, $T$ vaginalis, $N$ gonorrhoeae 6 ; bacterial vaginosis $11 ; C$ trachomatis 18 ). the instructions of the manufacturer of Chlamydiazyme ${ }^{r}$.

The diagnosis bacterial vaginosis was defined by at least three of the following four criteria in the absence of any other specific infection: homogeneous discharge, positive aminetest, $\mathrm{pH}$ greater than 4.5 and clue cells.

\section{Methods for comparison}

In cross tabulations the results of the cervical and vaginal specimens were compared concerning both micro-organisms. The findings in the posterior fornix were used as a reference in calculating the sensitivity of the cervical specimen. Assuming that culture does not give false positive results the specificity was considered to be $100 \%$.

\section{Results}

Study population

Eligible for the study were 692 patients of whom 10 women were unwilling to participate; 682 women were eventually enrolled in the study. Culture results of both the cervical and the vaginal swab were missing from six cases, results of the ELISA test for $C$.trachomatis were missing in 18 women. There was insufficient information from 11 women to exclude bacterial vaginosis.

In Amsterdam, 642 women (94\%) were admitted, $40(6 \%)$ in the east of the Netherlands. The mean and median age were respectively 28.9 and 27.2 years. The frequencies of $C$ albicans, $T$ vaginalis, $N$ gonorrhoeae, $C$ trachomatis and bacterial vaginosis are shown in table 1.

A cervical swab could not be taken, from eight patients because they had undergone a hysterectomy and in one patient a cervical swab only was available. Consequently 667 cases were included in the analysis.

\section{Candida albicans}

Infection due to a Candida species was found in either cervix or posterior vaginal fornix in 248 women $(37 \%)$. Of these infections 240 were due to $C$ albicans. In eight cases (3\%) other species were involved: Candida stellatoidea 3, Torulopsis glabrata 2, and in one patient each Candida krusei, Candida tropicalis and Candida guilliermondi. All yeast infections were included in the analyses and registered as $C$ albicans for the sake of convenience.

Table 2 compares the culture results from the cervix and vagina. In 14 cases of 247 vaginal positive women the culture of the cervical swab was negative for $C$ albicans. In

Table 2 Comparison of culture results of $C$ albicans from vaginal and cervical site

\begin{tabular}{llcl}
\hline & \multicolumn{3}{l}{ Posterior fornix } \\
\cline { 2 - 4 } & Positive & Negative & Total \\
\hline Cervix & & & \\
& & 1 & 234 \\
Positive & 233 & 419 & 433 \\
Negative & 14 & 420 & 667 \\
Total & 247 & & \\
Sensitivity of the cervical swab: $94 \%$ & \\
\hline
\end{tabular}


Table 3 Comparison of culture results of $T$ vaginalis from vaginal and cervical site

\begin{tabular}{lccc}
\hline \multicolumn{3}{c}{ Posterior fornix } \\
\cline { 2 - 4 } & Positive & Negative & Total \\
\hline Cervix & & & \\
& & 1 & 35 \\
Positive & 34 & 629 & 632 \\
Negative & 3 & 630 & 667 \\
Total & 37 & & \\
Sensitivity of the cervical swab: $92 \%$ & \\
\hline
\end{tabular}

one case $C$ albicans was only cultured from the cervix. Consequently, in $99 \%$ of the proven $C$ albicans cases, the yeast was found in the posterior fornix. In $95 \%$ C albicans was isolated from the cervical swab. In 652 of 667 women a concordant observation was made (98\%).

Trichomonas vaginalis

Thirty eight of $667(6 \%)$ women had a $T$ vaginalis cultured from either site (table 3 ). Three of $37(8 \%)$ vaginal positive cases had a negative culture from the cervix. In one woman $T$ vaginalis was only found in the cervical swab. Thus in $97 \%$ of the proven $T$ vaginalis cases the protozoon was found in the swab from the posterior fornix. In $91 \% T$ vaginalis was detected from the cervical swab. The sensitivity of the cervical swab was $92 \%$. In 663 cases the culture results from the two sites were concordant (99\%).

\section{Discussion}

In our study, the yield from the vagina was slightly better than that from the cervix, both for culture of $C$ albicans and $T$ vaginalis. On the other hand, the vaginal swab is not infallible as in single cases both organisms were detected only from the cervical swab.

We conclude that for screening purposes, the swab taken from the cervix for culture of $N$ gonorrhoeae can be reused for culture of $C$ albicans and $T$ vaginalis. This procedure does not appear to affect the sensitivity for the detection of gonorrhoea.

This information is relevant for general practitioners who want to take a specimen in women with vaginal symptoms but who may miss any specific sign of vaginitis or cervicitis. In an earlier study we observed that in approximately one quarter of these cases a specific cervical or vaginal infection could be found. In these cases the physican could confine himself to collecting one swab from the cervix, for detection of $C$ albicans, $T$ vaginalis and $N$ gonorrhoeae with minimal loss of sensitivity. Apart from this, a specimen from the cervix has to be obtained for diagnosis of $C$ trachomatis. However, if $T$ vaginalis and $C$ albicans are considered to be the most likely infections then the specimens should be collected from the vagina.

1 Finegold MT, Martin WJ. Diagnostic Microbiology. St Louis: The CV Mosby Company, 1982.

2 Rein MF. Trichomonas vaginalis. In: Mandell GL, Douglas RG, Bennett JE, eds. Principles and Practice of Infectious Diseases. 3rd ed. New York: John Wiley, 1990:2115-7.

3 Hook EW, Handsfield HH. Gonococcal infections in the adult. In: Holmes KK, Mardh PA, Sparling PF, Wiesner PJ, eds. Sexually Transmitted Diseases. 2nd ed. New York McGraw-Hill Book Company, 1990:149-65.

4 Warnock DW, Milne JD, Speller DCE. Vaginal candidosis and related infections. In: Hare MJ, ed. Genital Tract Infection in women. Edinburgh: Churchill Livingstone, 1988:199-215.

5 Fouts AC, Kraus SJ. Trichomonas vaginalis: Reevaluation of its clinical presentation and laboratory diagnosis. $\mathcal{F}$ Infect its clinical presentation

6 Sobel JD. Vulvovaginal Candidiasis. In: Holmes KK, Mardh PA, Sparling PF, Wiesner PJ, eds. Sexually Transmitted Diseases. 2nd ed. New York: McGraw-Hill Book Company, 1990:515-23.

7 Rein MF, Muller M. Trichomonas vaginalis and trichomoniasis. In: Holmes KK, Mardh PA, Sparling PF, Wiesner PJ, eds. Sxually Transmitted Diseases. 2nd ed. New York McGraw-Hill Book Company, 1990:481-92.

8 Rayner CFA. Comparison of culture media for the growth of Trichomonas vaginalis. $\mathrm{Br} f$ Venereal Dis 1968;44:63-6.

9 Lennette EH, Balows A, Hausler WJ, Shadomy HJ, eds. Manual of Clinical Microbiology. 4th ed. Washington DC American Society for Microbiology, 1985. 